Revista de Sociología

№ 19 - 2005

Facultad de Ciencias Sociales - Universidad de Chile

(p. 71 - 85)

\title{
DIVERSIDAD, REGULACIÓN Y UNIVERSIDAD PÚBLICA: TEMAS PARA UN DEBATE SOBRE LA EDUCACIÓN SUPERIOR EN CHILE
}

\section{Raúl Atria*}

\section{Definiendo el problema}

El concepto de universidad está experimentando cambios de reconocida importancia, debido, entre otros factores, al hecho de que no parece haber hoy en día consenso acerca de un modelo único o privilegiado respecto de esta institución. La clásica trilogía docencia, investigación y extensión como funciones esenciales que deben concurrir simultáneamente para conformar la universidad, ha quedado largamente sobrepasada por la extraordinaria variedad de respuestas adaptativas que las universidades han asumido, en el mundo moderno, para responder a los cambios acelerados de sus entornos tecnológicos, financieros y laborales. Universidades especializadas, universidades tecnológicas, universidades docentes son expresiones que de alguna manera las comunidades académicas y los analistas están usando para dar cuenta de esta diversidad. En el caso de Chile, a partir de la reforma de 1981 que abrió el campo de la actividad académica de la educación a la iniciativa del sector privado, se expandió considerablemente el número de instituciones que, en general adoptaron un modelo de concentración en un número limitado de áreas de formación disciplinaria y profesional y que optaron en la realidad, aunque no siempre en las declaraciones de principios o de misión institucional, por la función docente como eje principal de desarrollo de la institución. El grueso de las universidades privadas que nacieron a partir de la reforma de 1981, y algunas de las que se derivaron de las "tradicionales", han evolucionado como universidades eminentemente docentes y concentradas en un número reducido de áreas disciplinarias, conforme a la clasificación de la UNESCO. Al año 2003, había 61 universidades en el país, que pueden diferenciarse en los siguientes grupos ${ }^{1}$ : a) 9 tradicionales autónomas; b) 16 derivadas tanto de universidades estatales(13) como católicas (3); y c) 25 privadas.

\footnotetext{
${ }^{*}$ Director D epartamento de Sociología.

${ }^{1}$ De las nueve tradicionales 3 son estatales; 3 son públicas no estatales ubicadas en regiones (Austral, Concepción y F. Santa María) 2 católicas pontificas (Católica de Chile y Católica de Valparaíso) y 1 católica no pontifica (del Norte); de las 16 derivadas autónomas 13 son estatales y 3 católicas; de las 36 privadas 25 son autónomas certificadas como tales por el Consejo Superior de Educación, 8 en proceso de acreditación y 3 sujetas a régimen de examinación. (Schwember, 2005)
} 
Conforme progresa el proceso de creciente y sostenida diferenciación y diversificación institucional en los sistemas de educación superior, mayor es la gravitación que adquieren las universidades llamadas docentes. En esa misma medida arrecia el debate sobre el carácter de la misión universitaria y sobre el papel que el desarrollo del conocimiento no científico tiene en la configuración del modelo buscado y deseado de universidad. La ortodoxia sostuvo, con indiscutida convicción, que sin investigación no hay universidad posible. Sin embargo, la práctica está mostrando con la clásica porfía de la vida concreta, que no sólo es posible la universidad sin investigacion propia, sino que es, ademas, una forma deseable de concreción de modelos variados de universidad.

¿Como es la calidad de estas instituciones? Esta pregunta admite muchos matices, pero, en general se puede decir que aquellas instituciones que nacieron regidas por un proyecto académico nítido, con objetivos precisos y con un razonable respaldo económico, han ido afirmando su calidad a un tranco sostenido y tienen por tanto un buen pronóstico de calidad. Estas instituciones podrían ser englobadas bajo la noción de instituciones de "apropiación académica" en el sentido de que el proyecto de desarrollo respectivo y las políticas de gestión correspondientes, están fuertemente ligados a patrones que proviene de la cultura académica. Hay otras instituciones en el ámbito privado que son casos de "apropiación empresarial" donde el proyecto de desarrollo respectivo y su gestión, se enmarcan estrictamente dentro de la lógica de decisiones de negocios. En ambos casos se ha enfatizado la noción de apropiación para señalar que se trata de procesos que no necesariamente están atados a una determinada forma corporativa de propiedad privada de modo que los propietarios de la universidad pueden dar cabida a una u otra de las formas de apropiación señaladas. ${ }^{2}$

Es claro que las trayectorias de desarrollo de estos dos tipos de instituciones se proyectan hacia escenarios corporativos completamente diferentes. No es posible adentrarse en esta oportunidad en el análisis de esta dos trayectoria 0 modelos, pero tan sólo para marcar una indicación de esa diferencia, en el modelo de apropiación empresarial parece extremadamente posible que haya cabida para la maduración institucional endógena de procesos lentos, como son por ejemplo, la formación de masas críticas de académicos, o masas críticas de estudiantes que hagan posible la vida universitaria. D os parámetros que sirven para ponderar la calidad y la nitidez de los proyectos académicos son la calidad y dedicación del núcleo de académicos que imparten la docencia y la dotación y uso efectivo de recursos pedagógicos. O bviamente, si de universidades docentes se trata, lo primero que es necesario escrutar es la calidad de los insumos de la docencia (profesores,

\footnotetext{
${ }^{2}$ Esta noción de apropiación se aproxima más a la ideea de empoderamiento que de propiedad.
} 
DiVERSIDAD, REgULACIÓN Y UNIVERSIDAD PÚBLICA:

RAÚl Atria

bibliotecas, laboratorios). Dicho esto, se puede observar que las trayectorias recorridas por estas instituciones concentradas preferentemente en algunas áreas del conocimiento y de las profesiones y que están dedicadas a la docencia, han sido variadas, pero en general se pueden distinguir las siguientes rutas: a. universidades que accedieron a la autonomía; b. universidades en proceso avanzado de consolidación; c. universidades de desarrollo incipiente y d. universidades precarias.

El dato básico para partir es la heterogeneidad de las instituciones que forman parte de la estructura dela educación superior en Chile. Esta heterogeneidad

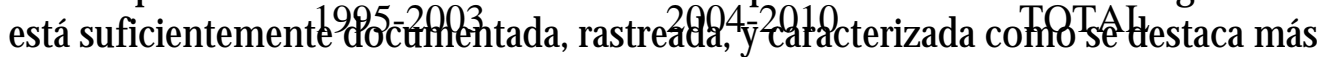

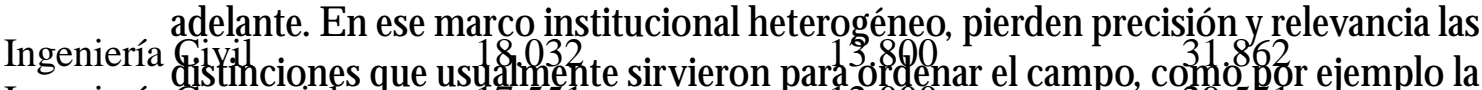
Ingeniería Comercia Educación Media

Derecho

Educación (1) 15 then

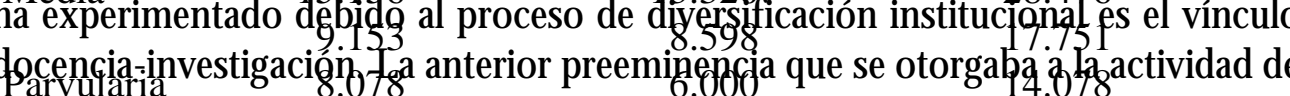

En essa tríada la relación gíue más cambios

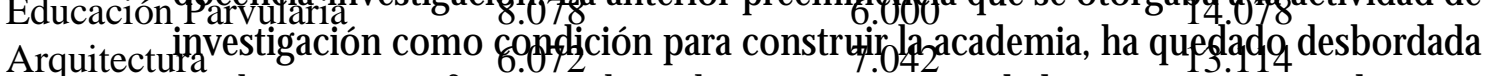

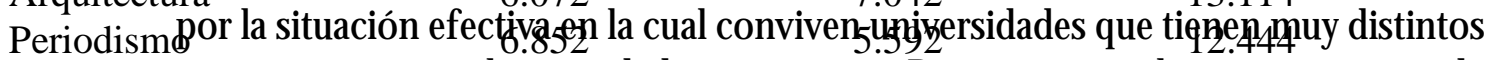

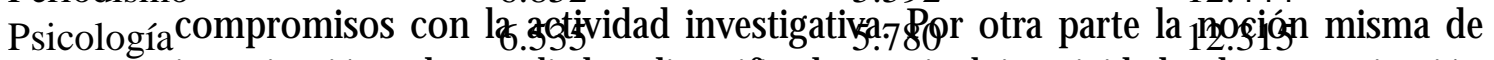
Agronomínnvestigación se ha amplizalo y diversificado pałäncluir actividades \&egenegrganización

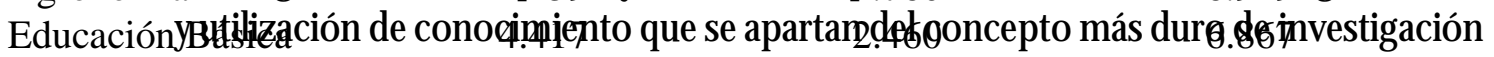
que no sólo incluye las actividades ya mencionadas sino que pone en el centro de la investigación la producción de conocimiento en los ámbitos disciplinarios y de frontera.

La heterogeneidad es un proceso irreversible que debe ser incorporado a la comprensión actual de la educación superior y al correspondiente diseño de opciones de política para contribuir a su desarrollo y afianzamiento. D esde el ángulo de las políticas no hay base alguna para sostener juicios negativos acerca de la 
heterogeneidad. Antes bien hay un desafío inevitable para potenciar las oportunidades que se abren con el proceso de diversificación, que deja entonces de ser una suerte de mal menor de la modernidad y se puede entender como una dinámica que acarrea con ella variadas posibilidades para fortalecer y ampliar el rango social y cultural de la educación superior y hacer de ésta una eficaz herramienta de desarrollo para los países.

\section{Rasgos del perfil del sistema}

\section{A. Elementos de diagnóstico}

D esde la reforma de 1981 a la fecha, el sector de la educación superior ha ido adquiriendo ciertas características que están relativamente consolidadas y que, en cierta forma, se tornan progresivamente irreversibles desde el punto de vista de las opciones de políticas de cambio para el sector. Existe hoy una acentuada diversificación del sistema que se expresa de diversas maneras:(i) diferenciación institucional (universidades - institutos profesionales - centros de formación técnica), (ii) régimen de propiedad (estatales - privadas), (iii) régimen de financiamiento (con aporte fiscal directo/ sin aporte fiscal directo), (iv) diferenciación funcional (universidades que hacen investigación/ universidades docentes), (v) localización geográfica (metropolitanas/ regionales), (vi) grado de complejidad (alto - medio bajo), (vii) tamaño (sobre 10.000 alumnos - bajo 400 alumnos), (viii) origen histórico (instituciones existentes antes de 1980; universidades derivadas de las universidades estatales; universidades creadas a partir de institutos profesionales derivados; universidades derivadas de universidades privadas con aporte fiscal; $\mathrm{y}$ instituciones propiamente privadas) ${ }^{3}$

Estas diferenciaciones no siempre significan cortes homogéneos, lo que permite establecer diversos tipos de categorías institucionales cruzando estos distintos factores de diferenciación (desde Universidades estatales y privadas con aporte fiscal directo, que hacen investigación de buen nivel, metropolitanas, de alta complejidad y gran tamaño; hasta CFT privados, sin aporte fiscal directo, netamente docentes, ubicados en regiones, que ofrecen una o dos carreras simples y tienen menos de 400 alumnos; pasando por diversas mezclas entre ambos extremos.

Para lograr que esta heterogeneidad se consolide como factor de diversidad estructural que contribuya así a fortalecer el sistema de educación superior, es preciso

${ }^{3}$ No deja de ser curioso que entre 1990 y 1995 se hayan creado más universidades con aporte fiscal que universidades privadasprivadas. 
DiVERSIDAD, REGULACIÓN Y UNIVERSIDAD PÚBLICA:

diseñar y aplicar políticas que establezcan la plena legitimidad institucional y social del desarrollo de instituciones en cada nivel, sin que existan incentivos encubiertos al desplazamiento hacia arriba desde uno a otro nivel. Este desplazamiento se refiere tanto a los Institutos Profesionales que se perciben a sí mismos como potenciales universidades, como a los Centro de Formación Técnica que se perciben como potenciales institutos profesionales. El desplazamiento hacia arriba también se refiere a los individuos que se perciben a sí mismos ( o son inducidos a ello por el marketing de las instituciones) como ocupantes temporales y transitorios (una especie de pasajeros por defecto), de cupos en los niveles inmediatamente "inferiores". Un factor decisivo para la consolidación y legitimidad de los tres niveles es el establecimiento de espacios de acción y de especialización efectiva de la oferta educacional de cada nivel.

No parece haber dudas en que la educación superior en Chile siempre ha sido profesionalizante. Sin embargo, la proliferación de carreras, y la mantención de esquemas rígidos en la mayoría de los casos (salvo los programas de bachillerato, potencialmente promisorios pero todavía en etapa de prueba, y de escaso peso en el conjunto) va en contra de las tendencias internacionales. El pregrado se mantiene sobredimensionado en las duraciones de las mallas curriculares, sobrecargado de una proliferación de contenidos y asignaturas, y contando cada vez con menos elementos académicos a pesar del agregado de la Licenciatura. D atos recopilados por Schwember muestran, por ejemplo, que entre los años 1995 y 2010 las estimaciones que se pueden hacer sobre la base de las cifras reales y actuales de matrícula, las universidades van a ser capaces de producir entre los años 1995 y 2010, las siguiente cifras de salida en las diez profesiones de mayor titulación en el sistema:

Como señala el autor, "no parece remotamente concebible que haya trabajo en campos con alguna afinidad a su entrenamiento para 6 mil arquitectos, o 18 mil ingenieros civiles, o para 6 mil psicólogos" (Schwember 2005:35). Es cierto que hay voces discrepantes respecto del potencial de empleabilidad para carreras como las indicadas, pero las proyecciones arriba expuestas, muestran que este tema no está zanjado y no puede dejarse livianamente entregado a la lógica de equilibrios de mercado. Esta aquí de verdad en juego la confiabilidad pública de las ofertas de carreras que se hacen al comienzo de los cinco o más años que serán invertidos por los estudiantes que se matriculan en las carreras. No parece justo que la pérdida de empleabilidad la hagan los estudiantes y sus familias.

\section{B. Necesidades en la definición de políticas}

Se requiere consagrar explícita e inequívocamente el reconocimiento de la diversidad estructural del sistema. Ello significa introducir, en el diseño e 
implementación de las políticas de educación superior, distinciones en algunas materias o dimensiones que parecen significativas para el reconocimiento de la diversidad, tales como:

-Rol de las universidades con aporte fiscal directo que implica introducir un principio de accountability indispensable para maximizar la pertinencia de las instituciones cuando hay fondos públicos comprometidos en ellas, independientemente del régimen corporativo que las caracterice. Introducir este principio de manera real y no puramente discursivo, obliga a revisar el concepto de autonomía institucional, que en nuestro medio ha dejado de lado toda connotación que apunte a la responsabilidad pública de las instituciones y ha terminado por ser un atributo perverso.

-E specificidad de las universidades estatales de modo que sobre ellas pueda construirse y desarrollarse un sistema universitario estatal, nacionalmente extendido, con amplia flexibilidad curricular y movilidad de estudiantes y académicos entre las instituciones y con diferenciación entre las universidades estatales de modo que puedan ellas tener, dentro del sistema estatal, una diferenciación de acuerdo a las áreas del conocimiento y de las profesiones donde hacen la apuesta por la máxima calidad.

-Rol de las universidades privadas propiamente tales (que no reciben directamente fondos públicos) por cuanto son estas instituciones las que al estar orientadas por el mercado en cuanto a los programas que ofrecen, introducen la determinación de la oferta de formación profesional desde el lado de la demanda y en cierto sentido producen así un efecto de arrastre que se extiende a otras instituciones en el conjunto diferenciado del sistema.

Es necesario tener en claro las expectativas del Estado con respecto a cada una de estas categorías, y los compromisos del Estado con cada una de estas categorías y/ o con sus alumnos. Para ello se necesita una cierta imagen socialmente aceptada del escenario futuro posible y probable del sistema y de sus componentes. La explicitación de una política de Estado respecto de la educación superior y de modo especial, respecto de sus propias universidades, es un elemento esencial para lograr esa visión del escenario futuro.

El reconocimiento de la diversidad estructural debe tener como correlato indispensable una especificación de los elementos (factores y mecanismos) de regulación aplicables a las distintas categorías de instituciones. Ello sin perjuicio de que existan políticas que establezcan procesos y modalidades de regulación de potencial aplicación general. ${ }^{4}$

\footnotetext{
${ }^{4}$ Los casos concretos son: a. el proceso de certificación de la autonomía institucional llevado a cabo por el Consejo Superior de Educación, y b. el proyecto piloto de acreditación institucional y de programas, conducido por la Comisión Nacional de Acreditación. (CNAP:2004).
} 


\section{Regulación y calidad}

RaÚl Atria

\section{A. Elementos de diagnóstico}

La situación actual, en materia de regulación es, aproximadamente la siguiente: (i) existe una inconveniente confusión de terminología regulatoria que se deriva de las normativas de 1981 y de 1990; (ii) coexisten mecanismos (estructuras y procedimientos) que no son equivalentes de regulación para un mismo fin; (iii) hay un limitado e insuficiente acopio y difusión de información pública acerca de los distintos componentes, ofertas y procesos, que perjudica la toma de decisiones individuales de los postulantes y usuarios de la educación superior; (iv) se reconoce la experiencia acumulada por el CSE respecto del nuevo sector privado de la educación superior y se advierte la presencia de señales positivas de receptividad del sector autónomo hacia esquemas de evaluación de calidad de resultados.

No existen verdaderamente mecanismos de regulación sistémica para las instituciones de educación superior y es un hecho que los gobiernos, desde 1990 no han sido capaces de modificar el marco legal respecto de la regulación, o no han estado de verdad interesados en subsanar deficiencias y confusiones que son reconocidas. Se trata de una situación que no podrá sostenerse en el tiempo, ya sea por presiones internacionales o por la necesidad de redefinir los mecanismos de asignación de recursos.

Es necesario diferenciar claramente entre mecanismos de regulación para instituciones no autónomas (que, a través de esos mecanismos adquieren la calidad de instituciones capaces de funcionar sin una supervisión estricta y cercana) y los que son aplicables a instituciones autónomas (en que la evaluación se hace más bien a través de la metaevaluación). Sin embargo, en las condiciones actuales del sistema, parece indispensable establecer condiciones previas para adscribirse al sistema de acreditación, aún en el caso de instituciones autónomas (sean éstas estatales, privadas con A porte Fiscal D irecto o privadas sin Aporte Fiscal Directo). Es importante reconocer que las señales positivas del sector autónomo hacia la regulación se emiten con cautela o debilidad (como por ejemplo la "autorregulación concordada en materia de carreras nuevas" en el seno de las instituciones del Consejo de Rectores, la "acreditación de programas de postgrado"), lo que puede atribuirse a las distintas "sensibilidades" derivadas de la heterogeneidad estructural del sistema.

El sistema de acreditación para instituciones autónomas tienen que estar regido por el Estado. En efecto, siendo que el objetivo básico de la acreditación institucional es dar garantía pública del grado en que las instituciones de educación superior cuentan con procesos eficaces para asegurar el cumplimiento de sus 
propósitos y objetivos y mejorar la calidad de los servicios que ofrecen, no parece posible ni conveniente que este proceso de evaluación no esté regido por una agencia pública y no se cómo una agencia de este tipo pudiera instalarse al margen del Estado. El hecho de que el proceso esté regido por el Estado, cualquiera sea la foram organizacional de la agencia del caso, no impide que puedan incorporarse distintos aspectos operativos, tales como: participación voluntaria pero con incentivos; autoevaluación con validación externa, aplicación de estándares comunes, según el área; establecimiento de un organismo acreditador de carácter técnico, autónomo y plural, que podría ser una extensión del $\mathrm{CSE}^{5}$; sistema extensible al pre y posgrado y a las nuevas ofertas educativas.

Apuntando en la dirección correcta, parece haber un amplio consenso que cruza toda la diversidad de instituciones que ya se ha mencionado, en que es conveniente asociar el acceso a ciertos recursos con la incorporación a mecanismos de acreditación (créditos o becas para estudiantes; fondos de investigación; fondos de desarrollo institucional, p. ej.). Es preciso considerar también la posibilidad de que toda asignación de recursos públicos vinculada con mecanismos de acreditación sea con exigencia de alguna proporción de recursos de contraparte y que esté dirigida específicamente a concretar acciones directamente derivadas de la acreditación 0 conectadas con ese proceso. ${ }^{6}$

\section{B. Necesidades de política para la acreditación}

Los gobiernos de la Concertación, han logrado incrementar significativamente el gasto público asignado al sistema de educación superior, lo que se ha manifestado en el perfeccionamiento y creación de nuevos mecanismos de financiamiento, permitiendo un mayor acceso a la educación superior. Sin embargo, este esfuerzo económico, no ha ido a la par con una política sustantiva que pretenda elevar los estándares de calidad del sistema. Es así como hoy se hace imprescindible dar un salto cualitativo en la educación superior y establecer

\footnotetext{
${ }^{5}$ En la discusión parlamentaria del proyecto de ley sobre el establecimiento de un sistema de aseguramiento de la calidad, se han delineado dos posibilidades: la creación de un nuevo ente supervisor o la introducción de cambios al actual CSE. Sin entrar a pronunciarse acerca de la conveniencia de adoptar una u otra posibilidad organizacional, puede sin embargo discutirse la conveniencia de extender la composición de ese ente a representantes de los poderes públicos y en tal sentido, no parece haber argumentos muy convincentes como para incluir representantes del Poder Legislativo en este ente. Tampoco parece convincente la ampliación de la representación del Ejecutivo más allá de la que hoy tiene a través del Ministro de Educación en el actual Consejo. Todo ello porque lo importante desde el punto de vista de la política de regulación, es que el ente regulador mantenga invariablemente su naturaleza pública autónoma.

${ }^{6}$ Respecto del establecimiento de mecanismos de regulación voluntarios, es preciso reconocer que hay cabida para levantar una serie de interrogantes, tales como ¿es posible mantener ese planteamiento desde la perspectiva de la coherencia sistémica? ¿se justifica que el proceso de acreditación sea voluntario, sobre todo en el caso de que las instituciones que reciben aportes del Estado? ¿es políticamente posible hacerlo obligatorio? ¿es conveniente establecer "notas" para los programas y carreras en términos de calidad, o sería preferible hacerlo en términos de cambios necesarios en los programas o carreras para mejorar o progresar?
} 
TEMAS PARA UN DEBATE SOBRE LA EDUCACIÓN SUPERIOR EN CHILE

RAÚl Atria

parámetros que regulen la calidad de la enseñanza impartida por las distintas instituciones.?

Respecto de criterios para la evaluación, todo el proceso de acreditación propuesto deberá estar regido esencialmente por los propósitos y definiciones institucionales. Sin embargo, y recordando siempre que son dichos propósitos y definiciones los que establecen el marco para la evaluación, ésta debe tomar en consideración el ajuste de la institución a ciertos criterios generales de calidad reconocidos internacionalmente como propios de toda institución de educación superior.

Para la efectiva aplicación de un sistema de acreditación eficaz, en el sentido general de la propuesta del proyecto de ley ya descrito, es necesario contar con un compromiso general y explícito del Estado de jugarse por la regulación, tanto en lo que se refiere a instalar efectivamente el sistema, como a incentivarlo y a financiarlo. En este sentido, la transición hacia un sistema único de supervisión, implicará mayores exigencias para el reconocimiento y funcionamiento de las instituciones, lo que parece adecuado. Las necesidades actuales de política no apuntan a una mayor desregulación del sistema, sino que por el contrario, hacia la legitimación de una nueva regulación. Si se reconoce la experiencia obtenida en la materia por la CNAP y el CSE, parecería lógico requerir de estos organismos un diseño de factibilidad de las funciones propuestas (regulación, evaluación, superintendencia e información pública) y de los mecanismos para transitar hacia un único sistema evaluativo de instituciones no autónomas.

Se necesitan definiciones de compromisos específicos del Estado respecto de qué se espera de ciertos tipos de instituciones. Sólo para los efectos de ilustrar este punto, se puede pensar, por ejemplo, un compromiso de no hacer proliferar las universidades chicas autónomas y estimular en vez su aglutinación corporativa para reforzar una escala de viabilidad de estas instituciones (tanto universidades como institutos y centro de formación técnica). La experiencia acumulada desde 1990, y el conocimiento de las efectos no anticipados, - 0 a veces perversos- de instrumentos de políticas que se han venido diseñando por agregación y sin una visión

${ }^{7}$ En marzo de 1999, por medio del decreto 51 del Ministerio de Educación, se crea la Comisión Nacional de Acreditación de Pregrado (CNAP), con la tarea de diseñar y proponer políticas de aseguramiento de la calidad para el conjunto de la educación superior del país. D icha comisión, en cumplimiento de la misión que le fue encomendada, presentó al Ministerio de Educación una propuesta para crear un "Sistema de aseguramiento de la calidad de la educación superior en Chile". Como resultado de ello, está en este momento en trámite parlamentario el proyecto de ley de establecimiento de un sistema nacional de aseguramiento de la calidad, preparado técnicamente por la Comisión Nacional de Acreditación. En el sistema de aseguramiento de la calidad en la forma definida por la CNAP, la acreditación institucional, se refiere a la evaluación de la existencia formal y la aplicación sistemáticade políticas y mecanismos que apunten al cumplimiento de los fines institucionales. De manera consistente con lo señalado más arriba, todas las instituciones deben abordar los siguientes dos aspectos centrales: a). mecanismos de gestión estratégica, para orientar el desarrollo de la institución desde su situación en un momento dado hacia un futuro deseado a mediano y largo plazo, y b). mecanismos para asegurar la calidad de la docencia de pregrado. 
relativamente coherente de las partes y de sus relaciones con el conjunto del sistema, permite poner legítimamente en la mesa de debates la cuestión de orientaciones explícitas que emanen del Estado respecto de qué se quiere promover y qué se quiere desincentivar. Por ejemplo, bien podría ser una de esas señales explícitas que hoy se echan de menos, el que se promueva derechamente la consolidación de los institutos profesionales y se desincentive, también derechamente, el paso de éstos a universidades.

D efinido un proceso de regulación que respete la "autonomía responsable" de las instituciones, deberá quedar en claro que esto no significa abdicar del derecho de la sociedad de obtener una cuenta pública del desempeño de todas las instituciones de educación superior. Regulación que respete la autonomía significa, de una u otra forma, el establecimiento de sistemas evaluativos para todas las instituciones, en los cuales se enfatice la preeminencia de la autoregulación. Respecto del ámbito de las instituciones nuevas, parece conveniente incrementar el tiempo mínimo bajo supervisión y que el status de autonomía responsable sea considerado luego de que, voluntariamente, las institución así lo solicite, a partir del cumplimiento previo de ciertos requisitos incluido un lapso mínimo de tiempo que habilite para esta solicitud.

Se requiere asimismo definir estándares de calidad diferentes según tipo de institución. Para emitirjuicios evaluativos parece adecuado considerar, como aspectos fundamentales, dos tipos de factores: (i) los que provienen de la misión y objetivos de cada institución, no necesariamente en las expresiones declarativas de la normativa formal sino de acuerdo a la manera como ellos son percibidos por los actores y los estamentos de las instituciones y (ii) los que provienen de estándares o indicadores propios de las áreas, disciplinas o profesiones involucradas. A estos dos ordenes de factores podría agregarse un tercero directamente vinculado con resultados, una vez que el sistema haya avanzado en el tratamiento diferenciado de los distintos tipos de instituciones que forman parte de él. Si esto se produce o se institucionaliza en el marco real de las políticas, entonces sería posible especificar estándares de calidad diferenciados según esos tipos de instituciones. Si el sistema da reconocimiento explícito a la diferenciación institucional, la ley pareja, en este sentido, puede ser muy dura.

El establecimiento de mecanismos de información pública completos, abiertos, actualizados y confiables, es imperioso para el buen funcionamiento del sistema en su conjunto. En las propuestas de políticas se incluyen medidas para mejorar la función de superintendencia y de información pública, estableciéndose, respecto de lo último, la consagración legal de la obligatoriedad de la información 
DiVERSIDAD, REGULACIÓN Y UNIVERSIDAD PÚBLICA:

RaÚl Atria

pública y los efectos de irregularidades en la provisión de información. Ello parece adecuado y necesario, pero, como ya se ha dicho, puede ser discutible la asociación directa de la información con juicios sobre la calidad.

\section{Hacia un modelo de universidad pública}

En el marco del nuevo perfil del sistema de educación superior en el país, el tema más crítico es de la regulación y la calidad de las instituciones del sistema. Se han dado pasos importantes para abordar este tema con enfoques y mecanismo apropiados para la diversidad que es propia del nuevo perfil.D entro de esta diversidad, sin embargo, no se ha abordado un tema que ha pasado a tener también un rasgo crítico para el conjunto del sistema y que es el del modelo de universidad pública, y dentro de ella la universidad estatal.

\section{A. Delineando el problema}

En general, se sabe relativamente bien cuál es el modelo de universidad que se ha implantado de manera predominante en el sector privado del sistema de educación superior para la formación profesional en el país. Se trata de un modelo coherente y claramente racional en su concepción, tiene definido o predefinido su estándar de calidad por la respuesta favorable del mercado. Ese modelo predominante tiene una expresión concreta en Chile que rige con claridad el conjunto de la oferta de carreras profesionales, es decir aquellas que están dirigidas a la inserción en el mercado laboral, que, en general, domina la educación superior en el nivel del pregrado. En este sentido, la regulación de esta oferta por el mercado, no sólo está afincada en las universidades privadas que surgieron con la reforma de 1981, sino que también alcanza a las universidades públicas, que están de hecho ajustando su oferta profesional con criterios de mercado.

Sostenemos, en primer lugar, que hay otras áreas de la actividad de las universidades que en la realidad no están regidas por esta lógica mercantil como son por ejemplo, los programas de posgrado, donde una parte importante de las maestrías, aquellas que se ha dado en llamar maestrías académicas para diferenciarlas de las profesionales (como es el caso, por ejemplo, de los MBA), no están sólo orientadas por criterios de mercado. Asimismo en el áreas de la investigación con impacto disciplinario, tampoco rige en plenitud la lógica del mercado. Este tipo de investigación y en general el posgrado, son ámbitos donde se instalan procesos de maduración lenta que no responden bien a la necesidades del corto plazo que rigen el ajuste por el mercado. Sostenemos, en segundo lugar, que es el campo de la 
universidad pública donde sería posible dar plena cabida a los ámbitos de la actividad universitaria que caen fuera del alcance del ajuste de la "oferta" académica por el mercado.

En nuestro medio, las universidades institucionalmente públicas no son todas estatales, pero una parte muy significativa de ellas, sí lo son, de manera que un avance en el sentido de delinear con mayor nitidez un "modelo" apropiado de universidad estatal, tendría un impacto señero en cuanto a la evolución de todo el sistema de educación superior. Es un hecho que no se ha producido todavía en Chile un modelo actualizado que realmente cautele y potencie el carácter público de la universidad estatal. Es cierto que a este requerimiento respondió la iniciativa del Ejecutivo de elaborar, en 1995, un proyecto de ley marco para la universidad estatal. Sin embargo, la apreciación sobre ese proyecto que terminó por prevalecer entre los actores de ese momento fue de insuficiencia, porque, si bien es cierto proporcionaba algunos elementos para ciertas definiciones que no estaban hasta este momento en la discusión normativa, fue, sin embargo, una iniciativa legal que siguió dejando en suspenso la definición de un modelo de universidad pública. Ese proyecto fue desahuciado finalmente por falta de consenso y de apoyo. No obstante, es el intento más explícito de parte de las autoridades gubernamentales de la Concertación por definir un modelo de universidad pública estatal. Por lo mismo, la discusión sobre dicho modelo sigue abierta. ${ }^{8}$

No es frecuente que actores responsables sostengan que las universidades públicas, y mucho menos las estatales, en el contexto actual de Chile, tengan que regirse por los cánones neoliberales de la economía. Hay, sin embargo, un imperativo de política que significa para estas universidades tener que evolucionar hacia esquemas de autofinanciamiento. Allí está radicada hoy día la discusión acerca del nexo entre las concepciones ideales de la educación superior estatal y la operativa práctica de las políticas públicas pertinentes (Salas y Aranda, 2005:81-83)

En una universidad pública es indispensable que haya algunas formas más o menos orgánicas de participación. el tema de la participación lleva de la mano una serie de otras cosas relacionadas, como el resguardo del pensamiento crítico, la efectividad de regulaciones públicas, el constreñimiento normativo que hay en el sistema y el tema de las metas de la institución.

${ }^{8}$ El nuevo estatuto de la Universidad de Chile, que ha sido recientemente aprobado en su trámite legislativo, puede ser un paso de cierta significación en el desarrollo de un"modelo" de universidad estatal, pero ello va a depender de la forma concreta como dicho estatuto sea aplicado e implantado en el sistema. 
DiVERSIDAD, REGULACIÓN Y UNIVERSIDAD PÚBLICA: TEMAS PARA UN DEBATE SOBRE LA EDUCACIÓN SUPERIOR EN CHILE

RaÚl Atria

La visión de las metas institucionales, tiene que ver con la estructura de gobierno, en donde se combina el particularismo de los actores con los intereses generales de la institución, de manera que las metas tienen que ser el resultado de un proceso donde priman los intereses generales, cualquiera sea el mecanismo interno que la organización se dé a sí misma para expresar esos intereses. Hay variadas formas en que pueden operar los mecanismos de representación que conducen a una articulación donde primen los intereses corporativos de cada grupo. Lo que verdaderamente interesa al respecto, es cómo dotar a la Universidad de una estructura de gobierno, de participación y de gestión, que posibilite la expresión orgánica de los intereses generales para sus metas. Entonces, el tema de fondo no es tanto formular el decálogo de las metas, sino que discernir acerca de cuál es el camino para poder formularlas.

En este sentido vale la pena subrayar el carácter multidimensional de la participación. Existe una forma de participación que es expresiva de la identidad de los agentes, especialmente estudiantes; hay participación para la información; hay formas de participación que tienen que ver con la representación; hay participación contralora, para asegurar transparencia y oportunidad en la rendición de cuentas. El punto clave para las políticas de gobierno y de gestión es cómo distinguir estos matices y articularlos de manera orgánica en la institución.

Respecto de la gestión, muchos participantes en las discusiones universitarias enfatizan la necesidad de que las personas elegidas rindan cuenta, que la gestión sea eficiente en términos de los fines de la institución. Esta aproximación finalista permite gestionar la universidad como una organización que cumple metas, que llega a ciertos fines eficazmente (fines entendidos como "funciones": la docencia, la investigación, la formación de profesionales, el crecimiento de la cultura, la generación de espacios de debate).

\section{B. Hacia una agenda inicial}

A partir de las anteriores consideraciones y de los principales rasgos del perfil que ha venido tomando el sistema de educación superior en Chile a lo largo de las últimas décadas, una agenda inicial de discusión posible para propender a un modelo de universidad debiera dar cabida al menos a los siguientes:

Como principio general, el modelo de universidad pública que se requiere, es muy distinto del modelo de la década del 60 y de principios del 70. La economía de ese entonces crecía hacia adentro, las empre-sas no tenían competencia entre sí, 0 -tenían un mercado muy asegurado. Ahora la economía nacional se desenvuelve 
en un contexto distinto, con procesos de producción y servicios que están globalizados, con fuertes exigencias de competitividad para las empresas de modo que la universidad y las empresas, deben interactuar activamente.

Un tema crítico es lograr un nivel de financiamiento mínimo asegurado para las Universidades estatales, política que puede ser extensible al resto de las universidades pertenecientes al Consejo de Rectores. Para la determinación de ese nivel se tiene que considerar la dimensión social de la educación superior estatal, elemento de equidad que es consustancial a la misión de la universidad pública.

D iseño y aplicación de una clara política de bienestar que permita abordar de manera efectiva los problemas de permanencia de los alumnos en el sistema, que vaya más allá de los mecanismo de financiamiento de los costos de aranceles que están operando por medio de créditos o becas según el caso.

Autonomía responsable de la gestión universitaria que permita el aseguramiento de la calidad de los proceso y resultados de las instituciones, y que posibilite la operación de mecanismos para hacer exigible la responsabilidad por los recursos públicos que se reciban, en particular la oferta de carreras profesionales que debiera regirse por alguna forma de contrato de desempeño.

D esarrollo y aseguramiento de la capacidad de las universidades públicas para responder a los requerimientos de la economía nacional, que se sustentan en el aprovechamiento de la competitividad del país, que debe ahora orientar su crecimiento y desarrollo econó-mico mirando preferentemente hacia afuera, hacia los mercados externos.

Instalar en las universidades estatales, una estructura organizacional más eficiente, que haga más horizontales las jerarquías burocráticas de la organización, para que las cadenas de decisión sean menores y la gestión gane en agilidad.

Para los efectos de normar la participación, distinguir -en lo que se refiere a la administración global de las universidades- instancias en las cuales se discutan o se perfilen las tomas de decisiones que van a afectar a las instituciones en el largo plazo, de aquellas instancias en las cuales se genera la discusión, regulación, armonización y la solución de problemas de corto plazo. 
DiverSIDAD, REgUlaCión Y UNIVERSIDAD PÚBLICA: TEMAS PARA UN DEBATE SOBRE LA EDUCACIÓN SUPERIOR EN CHILE

RaÚl Atria

Referencias bibliográficas

CNAP, G uía para la E valuación Ex terna. Proyecto Piloto de A creditación Institucional, Santiago, O ctubre 2004Schwember, Herman, "El contexto de la acción del Estado en la Educación Superior", Revista E stado, G obierno y G estión Pública, Instituto de Asuntos Públicos, Universidad de Chile, Vol II, No. 6, Santiago de Chile, 2005.

Salas, Víctor y Aranda, Rodrigo, Razones de la Presencia del Estado en la Educación Superior, en Revista Estado, Gobierno y Gestión Pública, INAP, Vol II. No. 6, Santiago, 2005. 